\title{
La calidad y la reforma del sector de la salud en América Latina y el Caribe
}

\author{
Anna Gabriela Ross, ${ }^{1}$ José Luis Zeballos ${ }^{1}$ y Alberto Infante ${ }^{1}$
}

RESUMEN El mejoramiento de la calidad de los servicios básicos de salud, junto con la búsqueda de equi dad, eficiencia, sostenibilidad y participación social en la provisión de estos servicios, ha sido uno de los principios rectores de la reforma del sector de la salud desde la I Cumbre de las Amé ricas en 1994. En el presente artículo se presentan algunos conceptos básicos en relación con el tema, se revisa la situación de la calidad en los sistemas y servicios de salud de América La tina y el Caribe, y se analizan las principales tendencias regionales que muestran los progra mas de garantía de calidad. Por último se recomiendan medidas para mejorar la calidad de ma nera continua y sostenible.

En la I Cumbre de las Américas celebrada en Miami, Estados Unidos de América, en diciembre de 1994, los Gobiernos de la Región reafirmaron su compromiso de incorporar en sus procesos de reforma del sector de la salud mecanismos para garantizar el acceso equitativo a determinados servicios básicos de salud y de mejorar la calidad de esos servicios. Al año siguiente, la Organización Panamericana de la Salud (OPS), con la participación del Banco Mundial (BM), del Banco Interamericano de Desarrollo (BID) y de otras agencias internacionales, patrocinó una Reunión Especial sobre Reforma del Sector de la Salud donde se establecieron sus criterios rectores, basados en los principios de equidad, efectividad y calidad, eficiencia, sostenibilidad y participación social (1). Desde entonces, el

\footnotetext{
1 Programa de Organización de Sistemas y Servicios de Salud, Organización Panamericana de la Salud, Washington, D.C., Estados Unidos de América. Dirección postal: PAHO/OPS, 525 Twenty-third Street, N.W., Washington, D.C. 20037, Estados Unidos de América. Correo electrónico: rossanna@paho.org
}

mejoramiento de la calidad ha sido uno de los objetivos proclamados de las reformas del sector de la salud.

\section{CONCEPTOS BÁSICOS}

En términos generales, la calidad puede definirse como el conjunto de características de un bien o servicio que logran satisfacer las necesidades y expectativas del usuario o cliente (2). Independientemente del enfoque que se adopte, el tema ha venido cobrando creciente importancia, no solo en el sector privado productivo, sino también en los sectores públicos sociales.

En el ámbito de los sistemas y servicios de salud se suele aceptar que la calidad tiene dos grandes dimensiones que están relacionadas, aunque son diferentes: la calidad técnica, que desde la perspectiva de los prestadores busca garantizar la seguridad, efectividad y utilidad de las acciones en pro de la salud, así como la atención oportuna, eficaz y segura de los usuarios de los servicios; y la calidad percibida por los propios usuarios, que toma en cuenta las condiciones materiales, psicológicas, administrativas y éticas en que dichas acciones se desarrollan.

Refiriéndose a la calidad técnica, Avedis Donabedian afirmó que consiste en la aplicación de la ciencia y la tecnología médicas de un modo que rinda los máximos beneficios para la salud, sin aumentar de forma proporcional los riesgos (3). La calidad es, por consiguiente, la medida en que se espera que la atención suministrada logre el equilibrio más favorable entre los riesgos y los beneficios. El modelo conceptual de Donabedian abarca tres aspectos del control de la calidad: estructura, proceso y resultados (4-5).

Hasta hace poco, el énfasis se ponía principalmente en la estructura y en los aspectos de regulación, acreditación, certificación y licenciamiento. Sin embargo, en la actualidad los procesos suscitan mayor interés, sobre todo aquellos que se orientan a garantizar la seguridad de las intervenciones tanto médicas como quirúrgicas y a fomentar la evaluación de las tecnologías, el 
empleo de intervenciones médicas basadas en pruebas confirmatorias, la atención protocolizada, y el empleo de indicadores intermedios que permitan medir el rendimiento de los servicios.

En relación con los resultados, la definición del estado de salud se ha ampliado paulatinamente hasta abarcar el funcionamiento emocional y social del paciente, además de su funcionamiento físico. Se considera importante no solo prolongar la vida, sino también mejorar su calidad, aminorando el dolor y las minusvalías o disfunciones, procurando que el paciente pronto se encuentre en condiciones de regresar al trabajo o de desempeñar actividades sociales y personales útiles, y velando por su bienestar y satisfacción después del tratamiento. Asimismo, cada vez es mayor la atención a los efectos económicos directos e indirectos de todo este proceso.

En lo que respecta a la calidad percibida por el usuario, el interés se ha venido ampliando desde la mera percepción del trato recibido en el momento de la asistencia (que suele investigarse mediante encuestas post-hospitalización), hasta abarcar todos los aspectos del proceso de atención: provisión de información, capacidad de elección, gestión administrativa, hospedaje, continuidad de los cuidados entre niveles de atención, etc. De hecho, la calidad percibida (en su doble dimensión humana y administrativa) figura cada vez más como un factor que afecta y modula notablemente a la calidad técnica, tanto en términos de los procesos como de los resultados.

En los países de la Región, ha habido poca uniformidad en el uso de ciertos conceptos, especialmente el del licenciamiento (6), que en determinados casos se aplica a los establecimientos y en otros a los recursos humanos. La resultante dificultad de los países para compartir sus experiencias y documentación al respecto ha sido una de las razones por las cuales la OPS, junto con representantes del Programa de Garantía de la Calidad de la Agencia Estadounidense para el Desarrollo Internacional, celebraron en mayo de 1999 un foro con la participación de expertos de América Latina y el Caribe, Canadá y Estados Unidos.
Durante esta actividad se examinaron los principales conceptos, se dieron a conocer los programas y las actividades en desarrollo en los países, junto con sus resultados $\mathrm{y}$, finalmente, se emitieron conclusiones y recomendaciones, tanto para los Gobiernos como para los organismos internacionales que se interesan por el tema de la calidad (7).

La calidad también ha sido asunto prioritario en la iniciativa sobre las Funciones Esenciales de la Salud Pública, desarrollada por la OPS. Dichas funciones comprenden un conjunto de medidas que el Estado tiene el deber de garantizar a toda la población, como base para mejorar la práctica de la salud pública y fortalecer el liderazgo de la autoridad sanitaria en todos los niveles gubernamentales (8). En este contexto, se propone incluir los siguientes elementos en la función individual y colectiva denominada Garantía de la Calidad de los Servicios de Salud: 1) fomentar la existencia de sistemas permanentes de garantía de la calidad y crear un sistema para que los resultados de las evaluaciones efectuadas con ellos puedan monitorearse de forma continua; 2) facilitar el establecimiento de normas aplicables a las características básicas que deben tener los sistemas de garantía de la calidad, y supervisar su cumplimiento por parte de los proveedores de servicios; 3) fomentar un sistema de evaluación de tecnologías de salud que participe en la toma de decisiones de todo el sistema de salud; 4) utilizar la metodología científica para evaluar intervenciones de salud de diverso grado de complejidad; y 5) usar este sistema para mejorar la calidad de la provisión directa de los servicios de salud.

Históricamente, las acciones en pro de la calidad y los programas responsables de su garantía y mantenimiento se originaron y desarrollaron dentro de los hospitales, y no fue hasta época reciente que se extendieron a los establecimientos del nivel primario $\mathrm{y}$, por último, a los programas del ámbito comunitario. Este sesgo doble —más interés en la estructura que en los procesos y los resultados, y mayor concentración en los hospitales que en el nivel primario y en la salud comunitariaha limitado (y aún limita) los logros de los países en materia de calidad.

No obstante, algunos fenómenos importantes, entre ellos ciertos cambios en la actitud de los usuarios de los servicios — quienes son ahora más exigentes y poseen más información-, la creciente participación de los medios de comunicación, y una mayor conciencia ética por parte de los profesionales, obligan a los Gobiernos a impulsar programas para mejorar continuamente la calidad de los servicios. Estos programas han de orientarse no solo a los aspectos estructurales de los servicios, sino también al proceso de atención a la salud y, sobre todo, a los resultados que dicho proceso genera.

Estas acciones y programas de garantía de calidad deben desarrollarse en varios ámbitos del sistema de salud, desde el servicio clínico individual hasta la red nacional de establecimientos y servicios. Lo ideal es que entre todos ellos haya concordancia y algún grado de articulación; que contribuyan a una cultura de calidad difundida a lo largo y ancho del sistema de salud; y que fortalezcan el ejercicio de la función rectora de las autoridades de salud, que es una de las funciones centrales de los Gobiernos. De hecho, es al propio Estado, y en concreto, a las autoridades de salud, a quienes compete garantizar la calidad de los servicios. De ahí que sea indispensable desarrollar mecanismos permanentes para medir y evaluar (tanto cuantitativa como cualitativamente y desde los puntos de vista de los financiadores, aseguradores, prestadores y usuarios) la calidad de estos programas. Ello implica establecer estándares, tarea que encierra una dimensión técnica (pertinencia, especificidad y sensibilidad) y una dimensión política (aceptabilidad para los distintos actores involucrados).

\section{LA SITUACIÓN DE LOS PROGRAMAS DE CALIDAD EN AMÉRICA LATINA Y EL CARIBE}

Según la información contenida en los 20 Perfiles de los Sistemas de Servicios de Salud disponibles en octu- 
bre de 1999 (9), la situación de los programas de garantía de la calidad en América Latina y el Caribe no puede considerarse alentadora. En la tercera parte de dichos perfiles se examina la manera en que los procesos de reforma sectorial desarrollados por la mayoría de los países desde comienzos de la pasada década han impulsado mecanismos de acreditación para establecimientos y servicios, de mejoramiento de la calidad (en sus aspectos técnicos y según la percepción de los usuarios), y de evaluación de las tecnologías de salud, todo ello en el contexto de los contenidos de los programas de reforma sectorial y de sus resultados en términos de calidad.

Se proporciona información sobre la calidad o los programas de calidad en las reformas sectoriales de 14 países, aunque en un caso la información no permite afirmar que existe una política para mejorar la calidad. Respecto a las iniciativas orientadas a fomentar la calidad técnica, el análisis de los cinco países con información buena o aceptable revela que en dos de ellos aún no parecen existir mecanismos o instancias para fomentar la calidad de los servicios y que en tres sí hay instancias encargadas y normas nacionales. Las diversas iniciativas de control de calidad abarcan los siguientes aspectos: 1) capacitación del personal en esa área; 2) establecimiento de centros de gestión de calidad; 3) evaluaciones públicas con la participación de los diferentes agentes sociales, y 4) establecimiento de comités para el control de infecciones. En tres países hay actividades que no están bien especificadas.

Según el análisis, solamente un país dispone de pruebas concretas de que la reforma del sector de la salud puede haber contribuido a mejorar la calidad técnica, pues han mejorado por lo menos tres de los cinco indicadores seleccionados: porcentaje de establecimientos del nivel primario con comités de calidad activos; porcentaje de hospitales con comités de calidad activos; disponibilidad de medicamentos esenciales en los distintos niveles de atención; incidencia de infecciones hospitalarias, y porcentaje de pacientes a los que se entrega un informe de alta o asistencia.
Respecto a la calidad percibida, se cuenta con información específica en cuatro países sobre la disponibilidad de encuestas de satisfacción, servicios de atención al paciente y centros de información bilingüe para la población indígena; capacidad para elegir el médico familiar, y establecimiento de sistemas para responder a las quejas de los usuarios.

Según el análisis de los cinco países con información buena o aceptable, tres países parecen disponer de pruebas de que la reforma del sector de la salud puede estar contribuyendo a mejorar la calidad percibida, ya que mejoraron por lo menos cuatro de los seis indicadores seleccionados: posibilidad de que el usuario, independientemente de su capacidad de pago, seleccione al prestador de los servicios de atención primaria; porcentaje de establecimientos con programas activos para mejorar el trato al usuario; porcentaje de establecimientos con procedimientos específicos de orientación al usuario; porcentaje de centros de salud y hospitales que realizan encuestas para explorar la percepción u opinión de los usuarios; comisiones de arbitraje activas, y grado de satisfacción de los usuarios de los servicios de salud.

De 11 países que proporcionaron información sobre la acreditación de los establecimientos y servicios, solamente cuatro parecen tener sistemas de este tipo. En un caso ha habido una propuesta inicial que aún no se ha incorporado al sector de la salud y que todavía no constituye un requisito para contratar a los prestadores de servicios. En dos países aún se están analizando diferentes posibilidades; en otro, el proceso se inició, se detuvo y se relanzó recientemente, y en otros tres países la reforma del sector sanitario no ha impulsado la creación de procedimientos de acreditación o la asignación de instituciones responsables.

Se dispone de información sobre la gestión y evaluación de tecnologías en salud en el caso de 14 países. En seis de ellos no parecen haberse creado procedimientos o instituciones destinadas a estos fines, mientras que en los ocho restantes sí ha habido algún grado de implantación. Tres países cuentan con centros o agencias específicas encargadas de la gestión o evaluación de tecnologías; en un país existen normas sobre la importación y evaluación de tecnologías, y en otros dos existen propuestas iniciales o en fase de diseño. Finalmente, en otro país se han editado guías para la práctica clínica, junto con protocolos y pautas de tratamiento, y el seguro social ha incluido la evaluación de tecnologías entre sus prioridades.

\section{TENDENCIAS REGIONALES EN LOS PROGRAMAS DE GARANTÍA DE CALIDAD DE LOS SERVICIOS DE ATENCIÓN}

Los programas en América Latina y el Caribe han mostrado hasta ahora cinco tendencias regionales. La primera consiste en el desarrollo de procesos, con lo cual se pretende llevar a cabo una acción continuada y establecer estrategias integradas, en lugar de promover medidas y acciones aisladas y esporádicas.

La segunda tendencia es el desarrollo de una acción integrada que haga uso combinado y simultáneo de varias de las herramientas de calidad, como se observa claramente en los procesos de mejoramiento continuo. Estos procesos forman parte de los estándares exigidos por Estados Unidos y Canadá.

Como tercera tendencia se observa una mayor orientación hacia los usuarios, tanto en los procesos como en los resultados, con la población y otros actores asumiendo una mayor participación en todo el proceso de gestión de la calidad.

En cuarto lugar, se percibe la tendencia a adoptar una visión sistémica en la cual los hospitales dejan de ser el centro del sistema de salud. El pasado énfasis en los establecimientos se transforma ahora en un interés por el conjunto de la red de servicios.

Como quinta y última tendencia se observa la creciente búsqueda de indicadores de desempeño. Para cuantificar el desempeño y demostrarlo, es preciso contar con sistemas de información adecuados y con indicadores y estándares apropiados. 
Señalan las autoridades nacionales que cualquier programa de control de la calidad deberá incluir medidas preventivas y de protección del medio ambiente, las cuales hasta ahora han sido sumamente escasas. Se reconoce, además, que ninguna estrategia es suficiente por sí sola o intrínsecamente superior a otra. De ahí que cada país deba formular la combinación mejor adaptada a sus necesidades.

Además de las tendencias ya mencionadas, los países encaminan sus esfuerzos hacia algunos otros aspectos críticos de los procesos de mejoramiento de la calidad: 1) la defensa del papel de las autoridades de salud como garantes de la calidad de la atención dentro de los procesos de reforma sectorial; 2) la defensa del papel del usuario y de la población como participantes activos en los programas de calidad; 3) la calidad técnica como resultado de las mejoras en la efectividad y utilidad de las prestaciones de salud; 4) la evaluación permanente de los servicios y tecnologías; 5) la elaboración y revisión de los estándares de calidad por consenso; 6) la relación entre la calidad y las formas de pago a los prestadores y los incentivos al desempeño profesional; 7) la medida en que se complementan los esfuerzos de los subsistemas público y privado en pro de la calidad; 8) la importancia de los sistemas de información para fomentar la calidad; y 9) el efecto de las investigaciones sobre la calidad de los servicios de salud. Es muy probable que un esfuerzo sostenido en estas áreas permita a los países mejorar la eficiencia y efectividad de sus servicios de salud.

\section{LOS RETOS FUTUROS}

La garantía de calidad de los servicios de salud en América Latina y el Caribe plantea grandes dificultades, tanto para las autoridades de salud como para los otros actores del sector. Al mismo tiempo, ofrece a los países oportunidades interesantes no solamente para establecer alianzas estratégicas entre los diversos organismos, sino también para cooperar con las autoridades de otros países a fin de resol- ver las nuevas necesidades que surgen al paso (10).

El reto fundamental para las autoridades de salud en los albores del nuevo siglo es garantizar el acceso de toda la población a servicios de calidad cuyo costo pueda ser enfrentado por la sociedad. Para lograrlo, deberán definirse estrategias y acciones claras que integren los intereses de los diversos actores del sector de la salud. Además, resulta conveniente que las autoridades de salud y los otros actores involucrados exploren el tema de la calidad a fin de 1) definir y aclarar el concepto de calidad que se va a adoptar en los programas; 2) estudiar y dar a conocer la situación de la calidad de los servicios de salud en los países; 3 ) dar prioridad a las acciones que se deben llevar a cabo en el corto y mediano plazo; 4) lograr que las expectativas de los prestadores y de los usuarios sean compatibles con la disponibilidad de los recursos; 5) definir la manera de incorporar al usuario y a la ciudadanía en el proceso; 6) investigar la relación entre el costo y la utilidad de los servicios que se prestan sin atención a la calidad (es decir, el costo real de la falta de calidad); 7) definir los instrumentos —certificación, acreditación, licenciamiento, etc. - que van a utilizarse y a integrarse dentro de un programa; y 8) prestar atención desde un principio a los aspectos antropológicos y de género.

Las autoridades de salud son las responsables de uniformar los criterios para la implantación del concepto de calidad, los actores que deberán participar, los parámetros que deberán regir, los indicadores y estándares que se utilizarán, y los instrumentos más apropiados y compatibles con el tipo de proceso que se va a desarrollar. En este sentido, puede ser muy útil tener en cuenta cuatro principios básicos a la hora de establecer programas nacionales de garantía de la calidad (11): 1) que se orienten hacia la satisfacción de las necesidades y expectativas del paciente y de la comunidad servida, o sea, hacia la atención centrada en el paciente; 2) que se concentren en los sistemas y procesos de prestación de servicios; 3) que utilicen información para analizar la prestación de servicios y, 4) que alienten un enfoque de trabajo en equipo para resolver problemas y mejorar la calidad.

Además, para que un programa de garantía de la calidad dé buenos resultados, debe contar con reglamentos claros que faciliten su adopción y mantenimiento, así como su sostenibilidad financiera (porque se trata de inversiones a largo plazo que permitirán hacer un uso óptimo de los recursos que se utilicen) y social (mediante una amplia participación de los sectores público y privado, universidades, grupos comunitarios y colegios profesionales, entre otros). Debe contar, asimismo, con un programa de educación para el personal encargado de los programas y el resto del equipo, incluidas las autoridades políticas y gerenciales. Este tipo de programa debe orientarse a mejorar todas las actividades de los servicios - aun las que están dirigidas hacia la compra y el mantenimiento de equipos, medicamentos, instalaciones, etc.y no únicamente las que tienen relación directa con el paciente.

\section{ALGUNAS ACTIVIDADES PARA LA COOPERACIÓN INTERNACIONAL}

El reto para los organismos internacionales consiste en asumir el papel de un eficiente agente catalizador —estratégicamente entrelazado con los procesos de reforma sectorial vigentes-, lo cual implica desarrollar acciones en varias áreas y siempre dentro del marco de una acción conjunta en la que participen otros actores. Cada vez más, cualquier tipo de cooperación, sea o no reembolsable, lleva implícita (y a veces explícita) la necesidad de contar con programas de calidad. Esta es una tendencia que deberá ser fomentada y reforzada.

Desde el punto de vista de la OPS, los principales retos consisten en cumplir los mandatos de la Cumbre de Presidentes celebrada en Miami, Estados Unidos, en 1994; en cooperar con los países para que se observen los principios de la reforma sectorial, se reorienten los servicios hacia la promoción de la salud y la prevención de enfermedades, y se desarrollen modelos de atención integral con garantía de la calidad; 
y en establecer alianzas con otros organismos para mejorar el apoyo a los países en la búsqueda de la calidad.

A continuación se mencionan algunas estrategias nacionales que podrían ser apoyadas por los organismos internacionales:

En relación con las autoridades de salud:

- desarrollo de sistemas para la evaluación de los servicios de salud;

- impulso a la evaluación de las tecnologías de salud antes de su introducción o diseminación en el sistema de salud, así como de aquellas tecnologías que actualmente se usan, donde existan dudas sobre su efectividad;

- inclusión de criterios de calidad en las nuevas formas de pago a los prestadores de servicios;

- estrategias que garanticen la acreditación de los establecimientos y las redes de servicios;

- apoyo a la elaboración de protocolos de atención;

- difusión de la medicina basada en pruebas confirmatorias (evidencebased medicine), y

- apoyo al desarrollo de planes piloto para el continuo mejoramiento de la calidad en los establecimientos y servicios.

En torno a la difusión de experiencias:

- actividades para que se investiguen, estudien y difundan los resultados de los proyectos de garantía y mejoramiento de la calidad que estén en marcha, tanto en el nivel nacional como en el regional;

- establecimiento de un centro de información y análisis regional (Clear inghouse) (12) que sea de utilidad para las autoridades, los financiadores, los prestadores y los usuarios, y

- apoyo al intercambio de experiencias entre países por medio de un programa de visitas recíprocas.

\section{En materia de capacitación:}

- desarrollo de seminarios con el personal gerencial, a fin de fomentar el mejoramiento continuo de la calidad de los servicios de la red, dentro del proceso de reforma, y

- desarrollo de programas de educación continua sobre el manteni- miento y mejoramiento de la calidad de los servicios para todo el personal de la red de establecimientos, así como de metodologías para la evaluación de los servicios, con participación de la comunidad.

En torno al desarrollo de alianzas estratégicas:

- Formación de alianzas y mecanismos de coordinación dentro de cada país con el fin de apoyar el desarrollo de procesos para el mejoramiento continuo de la calidad de los servicios de salud.

Huelga decir que en el desarrollo de todo este proceso podrían surgir nuevas dificultades, las cuales deberán ser detectadas oportunamente a fin de poder hacerles frente en futuros programas. El mejorar la calidad de los servicios de salud es un proceso permanente en el que participan distintos actores. Claramente, se trata de un proceso imprescindible para conseguir la mayor equidad y eficiencia de los servicios, meta que representa un perenne desafío para los países y los organismos internacionales.

\section{REFERENCIAS}

1. Organización Panamericana de la Salud. La cooperación de la OPS ante los procesos de reforma del sector salud. Washington, D.C.: OPS; 1997.

2. Organización Panamericana de la Salud. Programa de garantía y mejoramiento de calidad de los servicios de salud en América Latina y el Caribe. Washington, D.C.: OPS; 1999.

3. Milton T. Lean and mean: the quality of care in the era of managed care. J Public Health Policy 1998;19(1):5-13.

4. Knight W. Managed care. Aspen, Colorado: An Aspen Publication; 1998.

5. Donabedian A. Explorations in quality assessment and monitoring. Ann Arbor, Michigan: Health Administration Press; 1985.

6. Organización Panamericana de la Salud. Memorias del Foro Regional sobre la Acreditación, Certificación y Concesión de Licencias dentro del marco de la Garantía de la Calidad y Programas de Mejoramiento en los Países de las Américas y el Caribe, celebrado en Bo- gotá, Colombia del 19 al 21 de mayo de 1999. Washington. D.C.: OPS; 1999.

7. Organización Panamericana de la Salud y Programa de Garantía de la Calidad de la Agencia Estadounidense para el Desarrollo Internacional. Principales conclusiones y recomendaciones del Foro Regional de Calidad, Bogotá, Colombia, 19-21 de mayo de 1999. Washington, D.C.: OPS; 2000.

8. Organización Panamericana de la Salud, Centros para el Control y la Prevención de Enfermedades, Centro Latinoamericano de Investigación en Sistemas de Salud. La salud pública en las Américas: instrumento para la medición de las funciones esenciales de la salud pública. Washington, D.C.: OPS,CDC, CLAIS 2000. (Documento técnico HSP/HSO).

9. Organización Panamericana de la Salud, Agencia Estadounidense para el Desarrollo Internacional, Partnership for Health Reform, Family Planning Management Development, The Data for Decision Making Project. Inicia- tiva de Reforma del Sector Salud. http:/www. americas.health-sector-reform-org

10. Organización Panamericana de la Salud. Líneas estratégicas del Programa de Cooperación Técnica en la Calidad de Servicios de Salud. Washington. D.C.: OPS; 1988.

11. Agencia Estadounidense para el Desarrollo Internacional, Programa de Garantía de la Calidad. Garantía de calidad de la atención de salud en los países en desarrollo: serie de perfeccionamiento de la metodología de garantía de calidad. Nueva York: USAID; 1997.

12. Organización Panamericana de la Salud. Informe sobre la Reunión Regional sobre Programas de Garantía de la Calidad de los Servicios de Salud en el Marco de las Reformas Sectoriales, Relatoría General, Buenos Aires, Argentina, 17 a 19 de noviembre de 1997. (Documento mimeografiado pendiente de publicación por la División de Sistemas y Servicios de Salud, OPS). 
ABSTRACT Improving the quality of basic health services, together with the search for equity, efficiency, sustainability, and social participation, has been one of the guiding principles of health sector reform initiatives ever since the I Summit of the Americas was held in

Quality and health sector reform in Latin America and the Caribbean
1994. This article addresses some basic concepts, examines the status of quality control within health systems and services in Latin America and the Caribbean, and analyzes the most important trends observed in the Region in the establishment of quality assurance programs. Finally, ways of improving and monitoring quality continuously and sustainably are recommended.

\title{
PROGRAMA DE FORMACIÓN EN SALUD INTERNACIONAL
}

Fecha límite para la presentación de las solicitudes: 31 de julio de cada año

Se invita a las personas interesadas a concursar en el Programa de Formación en Salud Internacional de la Organización Pan americana de la Salud (OPS/OMS). 日 Programa está dirigido a profesionales residentes de los Países Miembros de la OPS, de 35 años de edad o menos, que posean una Maestría en Salud Pública, o su equivalente, y tengan un mínimo de dos años de experiencia en servicios de salud, educación o investigación en salud pública. $日$ programa formativo se desarrolla sobre la base de la incorporación de los participantes al trabajo de la Organización, en una dinámica de trabajo-estudio durante un período de 11 meses. Para mayor información sobre el Programa o el formulario de inscripción, las personas interesadas deberán acudir a la Oficina de la Representación de la OPS/OMS en su país o al:

\author{
Programa de Formación en Salud Internacional \\ Programa de Desarrollo de Recursos Humanos \\ División del Desarrollo de Sistemas y Servicios de Salud \\ Organización Panamericana de la Salud (OPS/OMS) \\ 525 Twenty-third St, N.W. \\ Washington, D.C. 20037 \\ Estados Unidos de América \\ Teléfono: (202) 974-3592, fax: (202) 974-3612 \\ http://www.paho.org
}

\section{TRAINING PROGRAM IN INTERNATIONAL HEALTH 2001}

\section{Deadline for the applications: 31 July of every year}

Interested individuals are invited to apply for the Training Program in International Health of the Pan American Health Organization (PAHOWHO). The Program is directed towards professionals from PAHOMember Countries who are 35 years of age or under, possess a Master's degree in Public Health or its equivalent, and have a minimum of two years experience in health services, education, or research. The Program follows a work-study format in which the participants are incorporated into the work of PAHOHeadquarters for 11 months. For more information on the Program or to obtain an application, interested individuals should contact the PAHOWHO Representation Office in their country or:

\author{
Training Program in International Health \\ Human Resources Development Program \\ Division of Health Systems and Services Development \\ Pan American Health Organization \\ 525 Twenty-third St, N.W. \\ Washington, D.C. 20037 \\ United States of America \\ Telephone: (202) 974-3592, fax: (202) 974-3612 \\ http://www.paho.org
}

